CIRJE-F-1135

\title{
Cooperation in Revision Games and Some Applications
}

\author{
Yuichiro Kamada \\ University of California, Berkeley \\ Michihiro Kandori \\ The University of Tokyo
}

December 2019

CIRJE Discussion Papers can be downloaded without charge from:

http://www.cirje.e.u-tokyo.ac.jp/research/03research02dp.html

Discussion Papers are a series of manuscripts in their draft form. They are not intended for circulation or distribution except as indicated by the author. For that reason Discussion Papers may not be reproduced or distributed without the written consent of the author. 


\title{
Cooperation in Revision Games and Some Applications*
}

\author{
KAMADA, Yuichiro ${ }^{\dagger}$ and KANDORI, Michihiro \\ ${ }^{\dagger}$ Haas School of Business, University of California, Berkeley \\ $\ddagger$ Faculty of Economics, University of Tokyo
}

November 22, 2019

\begin{abstract}
The present paper provides some examples that illustrate how cooperation is achieved among rational and selfish agents when (i) they prepare their actions in advance and (ii) they have some opportunities to revise their actions. Specifically, we use the framework of revision games introduced by Kamada and Kandori (2019). To judge the sustainability of cooperation in the examples, we show and utilize a simple and useful lemma.
\end{abstract}

*This article is based on materials previously presented in our unpublished working papers Kamada and Kandori (2017a, 2017b). It is also related to the R.K. Cho Economics Prize lecture series at Yonsei University by the second author, who received the prize in 2017. We thank Drew Fudenberg, Brett Green, Terry Hendershott, Johannes Hörner, Barton Lipman, Stephen Morris, Takuo Sugaya, Satoru Takahashi, Xavier Vives, and the seminar participants at the Far East and South Asia Meetings of the Econometric Society, Evolution of Cooperation Conference at IIASA, Summer Festival of Game Theory at Stony Brook, Cowles Foundation Economic Theory Summer Conference, and seminars at numerous universities including Boston University, University College London, Harvard University, University of Oxford, and Yale University. Omair Butt, Emily Her, Douglas Hong, Rohan Tripathi, Tawney Warren, Lingxuan Wu, and Tanlong Wu provided excellent research assistance. The second author acknowledges the support of JSPS KAKENHI Grant Numbers JP25380224 and JP17K03617. 


\section{Introduction}

In some economic and social interactions, agents have opportunities to revise their actions before the actions are finally implemented. For example, before the opening time of stock markets, traders can submit and revise their orders until they are finally implemented at the opening time. In electoral campaigns, parties often revise their announced policies. In such a situation, agents can sometimes achieve a "cooperative" outcome that Pareto dominates the Nash equilibrium of the one-shot game. This possibility was captured by the stylized model of revision games, which was introduced by Kamada and Kandori (2019). The present paper provides some examples that illustrate how cooperation is achieved in revision games.

The first example is a "stationary" version of the revision game. In the revision game in Kamada and Kandori (2019), there is a prespecified deadline, which is when the actions are implemented. In contrast, the first example does not have any deadline, and prepared actions may be implemented at any moment of time. This example turns out to be isomorphic to the repeated game, and it shows that there is a close connection between revision games and repeated games. We then turn to the revision game of Kamada and Kandori (2019), i.e., the one with a deadline, and consider two applications, namely a good exchange game and price competition, where the unique static Nash equilibrium is inefficient. In the good exchange game, players have a dominant action which is not to provide any good to the opponent; however, providing positive amounts to each other can Pareto-dominate such a situation. In the price competition, colluding at a high price Pareto-dominates the Nash price profile in which prices are so low that there is no further incentive for undercutting.

In each of these two applications, we solve for the optimal trigger strategy equilibrium plan by applying the general characterization in Kamada and Kandori (2019) that uses differential equations. The formal definition of a trigger strategy equilibrium plan in the revision game is presented in the next section. We show that under the optimal plan, over time, the amount of exchange decreases, and the price falls. Furthermore, using the condition for cooperation that Kamada and Kandori (2019) identify - called the Finite Time Condition - we determine when cooperation

is possible. In the good exchange game, the possibility of cooperation depends on the behavior of marginal cost and benefit of cooperation at the Nash equilibrium. In the price competition, product differentiation is necessary and sufficient for nontrivial 
cooperation to arise. To obtain those results, we show and utilize a simple and useful lemma (Lemma 1) to judge the sustainability of cooperation.

\section{Revision Games}

In this section we recapitulate the general framework and the main result of Kamada and Kandori (2019).

Component game: Component game is a two-player normal-form game with players $i=1,2$. There is a common action set $A$ that is a convex subset (an interval) of $\mathbb{R}$. The payoff function is $\pi_{i}: A \times A \rightarrow \mathbb{R}$. We assume symmetry, i.e., $\pi_{1}\left(a, a^{\prime}\right)=\pi_{2}\left(a^{\prime}, a\right)$ for all $a, a^{\prime} \in A$.

Revision game: Time continuously runs from $-T(<0)$ to 0 . At time $-T$, two players simultaneously choose their actions. During time in $(-T, 0)$, there is a Poisson process with arrival rate $\lambda>0$, and at each arrival of the Poisson hit, two players simultaneously revise their actions, observing all the past events. At time 0 , the action profile that is chosen at the last Poisson arrival is implemented, and the corresponding payoff profile is realized.

Assumptions and the finite time condition: We impose the following six assumptions throughout the paper.

- A1: A unique pure symmetric Nash equilibrium action $a^{N}$ and the unique best symmetric action $a^{*}:=\arg \max _{a \in A} \pi(a)$ exist, and $a^{N}<a^{*}{ }^{1}$

- A2: The symmetric payoff $\pi(a)$ is strictly increasing for $a<a^{*}$.

- A3: $\pi_{1}\left(a_{1}, a_{2}\right)$ is continuous. Furthermore, $\max _{a_{1}} \pi_{1}\left(a_{1}, a_{2}\right)$ exists for all $a_{2}$, and therefore we can define the gain from deviation at a symmetric profile $(a, a)$ by

$$
d(a):=\max _{a_{1}} \pi_{1}\left(a_{1}, a\right)-\pi_{1}(a, a)
$$

\footnotetext{
${ }^{1}$ This inequality is without loss of generality, and the case with $a^{*}<a^{N}$ can be analyzed in a symmetric manner.
} 
- A4: The gain from deviation $d(a)$ is strictly increasing on $\left[a^{N}, a^{*}\right]$ and nondecreasing for $a^{*}<a$.

- A5: The gain from deviation $d$ (defined by (1)) is differentiable, and $d^{\prime}>0$ on $\left(a^{N}, a^{*}\right]$.

- A6: Function $f(x):=\frac{\lambda\left(d(x)+\pi(x)-\pi^{N}\right)}{d^{\prime}(x)}$ is Lipschitz continuous on $\left[a^{N}+\varepsilon, a^{*}\right]$ for any $\varepsilon \in\left(0, a^{*}\right]$, where $\pi^{N}:=\pi_{i}\left(a^{N}, a^{N}\right) .^{2}$

Also, the following condition is the key to distinguishing those component games with which a nontrivial equilibrium exists in the revision game and those with which there is no such equilibrium.

- Finite Time Condition

$$
\lim _{a \downarrow a^{N}} \int_{a}^{a^{*}} \frac{1}{f(x)} d x<\infty .
$$

Optimal trigger strategy equilibrium plan: Under a trigger strategy equilibrium, cooperation is sustained in the following manner: on the path of play, players prepare an action prescribed by a plan $x(t)$. Namely, when a revision opportunity arrives at time $t$, players are supposed to revise their actions to $x(t)$. If any player deviates from this instruction, both players revert to a Nash action in all future revision opportunities. The plan $x(t)$ has to be such that players prefer following it to deviating to a static best response. As a consequence of this incentive constraint, under condition $(2), x(t)$ starts with the fully collusive level (when the time to the deadline is sufficiently long) and gradually tends to the Nash action as the time $t$ approaches the deadline.

A trigger strategy is characterized by its revision plan $x:[0, T] \rightarrow A$. Players start with initial action $x(T)$, and when a revision opportunity arrives at time $-t$, they choose action $x(t)$. If any player fails to follow that rule, then both players choose the Nash equilibrium action of the component game in all future revision opportunities. Formally, the set of feasible plans is:

$$
X:=\{x:[0, T] \rightarrow A \mid \pi \circ x \text { is measurable }\} .
$$

\footnotetext{
${ }^{2} \mathrm{~A}$ function $f$ is Lipschitz continuous on $\left[a^{N}+\varepsilon, a^{*}\right]$, if there exists a finite number $K \geq 0$ such that $\left|\frac{f(x)-f(y)}{x-y}\right| \leq K$ for all $x \neq y$ in $\left[a^{N}+\varepsilon, a^{*}\right]$.
} 
Given a feasible plan $x \in X$, the (trigger strategy) incentive constraint at time $t$ is

$$
(\mathrm{IC}(\mathrm{t})): \quad d(x(t)) e^{-\lambda t} \leq \int_{0}^{t}\left(\pi(x(s))-\pi^{N}\right) \lambda e^{-\lambda s} d s
$$

The set of trigger strategy equilibrium plans is:

$$
X^{*}:=\{x \in X \mid \mathrm{IC}(\mathrm{t}) \text { holds for all } t \in[0, T]\} .
$$

A plan that achieves the highest ex ante expected payoff within $X^{*}$ is referred to as an optimal trigger strategy equilibrium plan. The following result claims uniqueness of such a plan. In fact, if a plan $x$ is an optimal trigger strategy equilibrium plan, then another plan $y$ that does not coincide with $x$ only for $t$ 's in a measure zero set also constitutes an optimal trigger strategy equilibrium plan. The uniqueness that we state below is modulo such multiplicity. ${ }^{3}$

Theorem 1 (Kamada and Kandori (2019)). Suppose that A1-A6 hold.

1. The optimal trigger strategy equilibrium plan $\bar{x}(t)$ is the unique plan with the following properties: (i) it is continuous in $t$ and departs $a^{N}$ at $t=0$ (i.e., $\bar{x}(t)=a^{N}$ if and only if $t=0$ ), (ii) for $t>0$, it solves differential equation $\frac{d \bar{x}}{d t}=f(\bar{x}(t))$, where

$$
f(x):=\frac{\lambda\left(d(x)+\pi(x)-\pi^{N}\right)}{d^{\prime}(x)}
$$

until $\bar{x}(t)$ hits the optimal action $a^{*}$, and (iii) if $\bar{x}(t)$ hits the optimal action $a^{*}$ it stays there (i.e., $\bar{x}\left(t^{\prime}\right)=a^{*}$ for some $t^{\prime} \leq T$ implies $\bar{x}\left(t^{\prime \prime}\right)=a^{*}$ for all $\left.t^{\prime \prime} \in\left[t^{\prime}, T\right]\right)$.

2. The plan $\bar{x}(t)$ is nontrivial (i.e., $\bar{x}(t) \neq a^{N}$ for some $t$ ) if and only if the Finite Time Condition (2) holds. Under the Finite Time Condition (2), if the time horizon $T$ is large enough, $\bar{x}(t)$ always hits the optimal action $a^{*}$ at a finite time

$$
t\left(a^{*}\right):=\lim _{a \downarrow a^{N}} \int_{a}^{a^{*}} \frac{1}{f(x)} d x .
$$

3. If $\liminf _{a \downarrow a^{N}} \frac{d(a)}{\pi(a)-\pi^{N}}>0$, the Finite Time Condition fails and the unique trigger strategy equilibrium is to play the Nash action all the time: $x(t) \equiv a^{N}$.

\footnotetext{
${ }^{3}$ See Proposition 1 in Kamada and Kandori (2019) for the detail.
} 
Part 2 of Theorem 1 implies the following lemma that we utilize in the three examples in the next section. Since $\frac{1}{f(x)}$ in the definition of the Finite Time Condition (2) is finite for $x \in\left(a^{N}, a^{*}\right]$, the following holds.

Lemma 1. If $\lim _{a \downarrow a^{N}}\left|\frac{1}{f(a)}\right|$ exists and is finite, the Finite Time Condition (2) holds and the optimal trigger strategy equilibrium plan $\bar{x}(t)$ is nontrivial (i.e., not identically equal to the Nash action $a^{N}$ ).

This lemma will be useful in judging sustainability of cooperation in applications. This is because, in many cases, we can use l'Hôpital's rule to show that the limit of $\left|\frac{1}{f(a)}\right|$ as $a \downarrow a^{N}$ is well-defined and is finite.

\section{An Example (Two Samurai): Stationary Revi- sion Games}

Revision games refer to a class of games where (i) a normal-form game is played only once, (ii) players must prepare their actions in advance, (iii) the opportunities to revise prepared actions arrive randomly over time, and (iv) prepared actions are observable. Furthermore, there is a prespecified deadline at which the actions are implemented. In this section, we look at an example where there is no deadline, while keeping all the assumptions (i) - (iv). In the example, the problem is stationary in the sense that in each period $t=0,1,2, \ldots$, there is a fixed, positive probability $p$ with which a given normal-form game is played. We refer to such a class of games as stationary revision games. This class will turn out to be isomorphic to a familiar class of games, and it helps to build some intuition on how revision games work. The point we make is a simple one, so we just present an example.

Suppose that a rural village faces an attack of bandits. In each period $t=$ $0,1,2, \ldots$ the bandits attack the village with probability $p \in(0,1)$ around midnight. They attack only once. The villagers hired two samurai, $i=1,2$, and they must prepare to defend the village (to show up at the village gate around midnight) or not (to hide away and watch the gate from a distance). Hence in each period the two samurai observe each other's prepared actions. When the bandits attack, the samurai receive the following payoffs. 


\begin{tabular}{|c|c|c|}
\hline & Defend & Hide \\
\hline Defend & 2,2 & $-1,3$ \\
\hline Hide & $3,-1$ & 0,0 \\
\hline
\end{tabular}

This is a Prisoner's Dilemma game. Now consider player $i$ 's expected payoff. We denote player $i$ 's payoff by $\pi_{i}(t)$, when the bandits' attack occurs at time $t$. We also assume that players have a common discount factor $\delta \in(0,1)$. Player $i$ 's expected payoff is

$$
p \pi_{i}(0)+\delta(1-p) p \pi_{i}(1)+\delta^{2}(1-p)^{2} p \pi_{i}(2)+\cdots=p \sum_{t=0}^{\infty} \bar{\delta}^{t} \pi_{i}(t)
$$

where $\bar{\delta}:=\delta(1-p)$. Therefore, stationary revision games are isomorphic to infinitely repeated games, and cooperation can be sustained in a subgame perfect equilibrium if $\delta$ is high and $p$ is small. ${ }^{4}$ Even though the prisoner's dilemma game is played only once, players manage to cooperate. A mechanism to sustain cooperation is the trigger strategy, which works as follows. As long as the samurai have been showing up at the gate, they continue to do so (to prepare to defend the village). If anyone hides away, however, they stop showing up at the gate in the future.

\section{Applications}

In this section, we use the general framework of revision games (with a prespecified deadline) to analyze some economic applications. Specifically, we use the differential equation provided in Theorem 1 to analyze good exchange games and price competition with product differentiation.

\subsection{Good Exchange Game}

We first present a simple model to illustrate how a revision game works. Suppose two players produce and exchange goods. Player $i$ produces $a_{i}$ units of goods, with

\footnotetext{
${ }^{4}$ Sherstyuk, Tarui, and Saijo (2013) independently make the same observation about the equivalence of repeated games and stationary revision games.
} 
production cost $c\left(a_{i}\right)$ and gives it to player $-i$, who enjoys benefit $b\left(a_{-i}\right){ }^{5}$ Formally, this component game has two players $i=1,2$ with a common action space $A=[0, \bar{a}]$ for some $\bar{a}>0$, and their payoff function is

$$
\pi_{i}\left(a_{i}, a_{-i}\right)=b\left(a_{-i}\right)-c\left(a_{i}\right)
$$

where $b$ and $c$ are twice continuously differentiable and strictly increasing functions such that $b(0)=c(0)=0$ and $b^{\prime}(0)>0$. Note that there is a dominant strategy Nash equilibrium action $a_{i}=0$ and the Nash payoff is $\pi^{N}=0$. We assume that the Nash equilibrium is inefficient, and there exists a unique optimal action $a^{*}>0$ that maximizes the symmetric payoff $\pi(a)=\pi_{i}(a, a)$. We also assume that $\pi(a)$ is strictly increasing on $\left[0, a^{*}\right]$. With these assumptions, A1-A6 are satisfied. Let us call this class good exchange games.

The differential equation for the optimal trigger strategy equilibrium plan is

$$
\frac{d x}{d t}=f(x)=\lambda \frac{b(x)}{c^{\prime}(x)}
$$

This is derived as $f(x):=\frac{\lambda\left(d(x)+\pi(x)-\pi^{N}\right)}{d^{\prime}(x)}=\frac{\lambda(c(x)+(b(x)-c(x))-0)}{c^{\prime}(x)}$.

When is cooperation sustained? For example, the public goods provision game, where

$$
\pi_{i}\left(a_{i}, a_{-i}\right)=\left(a_{i}+a_{-i}\right)-r a_{i}, \quad 1<r<2, \quad a_{i}, a_{-i} \in[0, \bar{a}]
$$

can be regarded as a special case of the good exchange game with $b\left(a_{-i}\right)=a_{-i}$, $c\left(a_{i}\right)=(r-1) a_{i}$ and $a^{*}=\bar{a}$. Part 3 of Theorem 1 implies that no cooperation is sustained in this special case. The impossibility of cooperation comes from the property that $c^{\prime}(0)>0$ (the Nash action $a^{N}=0$ is a corner solution) and $b^{\prime}(0)$ is finite. More generally, the good exchange game provides the following insights into when cooperation is sustainable.

Proposition 1. In the revision game of the good exchange game, the possibility of cooperation depends on the marginal cost and benefit at the Nash action:

1. When $c^{\prime}(0)=0$ and $c^{\prime \prime}(0)>0$, there exists a nontrivial trigger strategy equilibrium plan.

\footnotetext{
${ }^{5}$ Alternatively, we can assume that players produce and exchange one unit of goods, and $a_{i}$ represents the quality of goods produced by player $i$.
} 
2. When $c^{\prime}(0)>0$, there does not exist a nontrivial trigger strategy equilibrium plan if $b^{\prime}(0)<\infty$.

3. When $c^{\prime}(0)>0$, there is $b(\cdot)$ with $b^{\prime}(0)=\infty$ such that there exists a nontrivial trigger strategy equilibrium plan.

Proof. The Finite Time Condition (2) is expressed as

$$
\lim _{a \downarrow a^{N}} \int_{a}^{a^{*}} \frac{1}{f(x)} d x<\infty \Longleftrightarrow \lim _{a \downarrow a^{N}} \int_{a}^{a^{*}} \frac{c^{\prime}(x)}{\lambda b(x)} d x<\infty .
$$

This is satisfied in Case (1). Since $\frac{c^{\prime}(x)}{b(x)}<\infty$ for any $x>0$, and $\lim _{x \downarrow a^{N}} \frac{c^{\prime}(x)}{b(x)}=$ $\lim _{x \downarrow a^{N}} \frac{c^{\prime \prime}(x)}{b^{\prime}(x)}<\infty$ by l'Hôpital's rule. Hence, Lemma 1 implies that cooperation can be sustained by the optimal trigger strategy equilibrium plan. In Case (2), the sufficient condition for no cooperation (part 3 of Theorem 1)

$$
\lim _{x \downarrow a^{N}} \inf \frac{d(x)}{\pi(x)-\pi^{N}}>0
$$

is satisfied. This is because $\frac{d(x)}{\pi(x)-\pi^{N}}=\frac{c(x)}{(b(x)-c(x))-0}$ and by l'Hôpital's rule

$$
\lim _{x \downarrow 0} \frac{c(x)}{b(x)-c(x)}=\frac{c^{\prime}(0)}{b^{\prime}(0)-c^{\prime}(0)}>0 .
$$

In Case (3), cooperation is sustained if, for example, $b(x)=\sqrt{x}$ and $c(x)=x$. The integrand $\frac{1}{f(x)}=\frac{c^{\prime}(x)}{\lambda b(x)}=\frac{1}{\lambda \sqrt{x}}$ in the Finite Time Condition (2) diverges to infinity as $x$ tends to the Nash action 0, but it does so slowly enough in this example. As a result, the Finite Time Condition (2) holds because

$$
\lim _{a \downarrow 0} \int_{a}^{a^{*}} \frac{1}{f(x)} d x=\left[\frac{2}{\lambda} x^{\frac{1}{2}}\right]_{0}^{a^{*}}=\frac{2}{\lambda} a^{* \frac{1}{2}}<\infty,
$$

so the cooperation is sustained by the optimal trigger strategy equilibrium plan.

We present a simple example that admits a closed-form solution, and lets us evaluate how much cooperation can be sustained.

\section{$\underline{\text { Linear benefit and quadratic cost }}$}




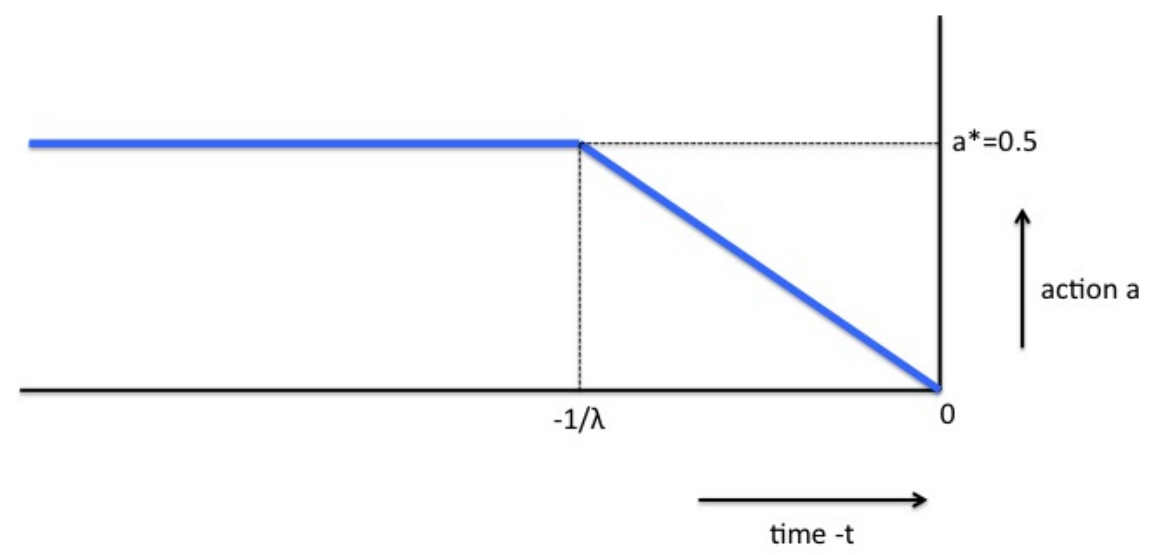

Figure 1: The optimal trigger strategy equilibrium plan $\bar{x}(t)$ for the good exchange game.

Proposition 2. In the revision game of the good exchange game with $b(a)=a$ and $c(a)=c \cdot a^{2}$ where $c>0$ is a constant, the optimal trigger strategy equilibrium plan, $\bar{x}(t)$, is characterized by

$$
\bar{x}(t)=\left\{\begin{array}{ll}
\frac{\lambda}{2 c} t & \text { if } t<t\left(a^{*}\right) \\
a^{*}=\frac{1}{2 c} & \text { if } t\left(a^{*}\right) \leq t
\end{array},\right.
$$

where $t\left(a^{*}\right)=\frac{1}{\lambda}$.

The plan characterized in Proposition 2 is depicted in Figure 1 for the case with $c=1$.

When $T \geq 1 / \lambda$, the plan starts at the optimal action $a^{*}=0.5$ and stays there until the time reaches $-1 / \lambda$. After that, the prepared action decreases over time to reach the Nash action $a^{N}=0$ at the deadline. The closed-form solution of the optimal trigger strategy equilibrium plan enables us to compute the expected payoff. Specifically, the next corollary shows that $74 \%$ of the fully collusive payoff can be sustained through the revision process, even though the players do not have a long- 
term relationship.

Corollary 1. In the revision game of the good exchange game with $b(a)=a$ and $c(a)=c \cdot a^{2}$ where $c>0$ is a constant, for any $\lambda>0$ and $T>t\left(a^{*}\right)=\frac{1}{\lambda}$, the following are true, where e denotes the base of natural logarithms:

1. The expected payoff under the optimal trigger strategy equilibrium plan is $\frac{1}{2 e c}$.

2. The ratio of the expected payoff under the optimal trigger strategy equilibrium plan to the fully collusive payoff is $\frac{2}{e} \cong 0.74$, independent of the value of $c$.

Proof. Part 1: The expected payoff under the optimal trigger strategy equilibrium can be calculated as follows:

$$
\begin{gathered}
\int_{0}^{t\left(a^{*}\right)}\left(\bar{x}(t)-c \bar{x}(t)^{2}\right) \lambda e^{-\lambda t} d t+e^{-\lambda t\left(a^{*}\right)}\left(a^{*}-c \cdot\left(a^{*}\right)^{2}\right) \\
=\int_{0}^{\frac{1}{\lambda}}\left(\frac{\lambda}{2 c} t-c\left(\frac{\lambda}{2 c} t\right)^{2}\right) \lambda e^{-\lambda t} d t+e^{-\lambda \frac{1}{\lambda}}\left(\frac{1}{2 c}-c \cdot\left(\frac{1}{2 c}\right)^{2} \cdot\right)=\frac{1}{2 c e} .
\end{gathered}
$$

Part 2: The fully collusive payoff is $\frac{1}{2 c}-c \cdot\left(\frac{1}{2 c}\right)^{2}=\frac{1}{4 c}$. Thus, the ratio of these two valued is equal to $\frac{\frac{1}{2 c e}}{\frac{1}{4 c}}=\frac{2}{e} \cong 0.74$.

\subsection{Price Competition: Product Differentiation Affects Col- lusion}

We consider the price-competition revision game, which captures the situation where firms revise their posted prices before the opening of the market/their stores. We will show that firms' abilities to collude hinges on the degree of product differentiation. In particular, we show that product differentiation is a necessary and sufficient condition for the sustainability of collusive prices. This prediction is in stark contrast to the prediction of infinitely repeated games, in which for any level of product differentiation, sufficient patience guarantees sustainability of collusion.

To demonstrate this result, we need a model to accommodate various degrees of product differentiation, including no differentiation as a special case. A standard 


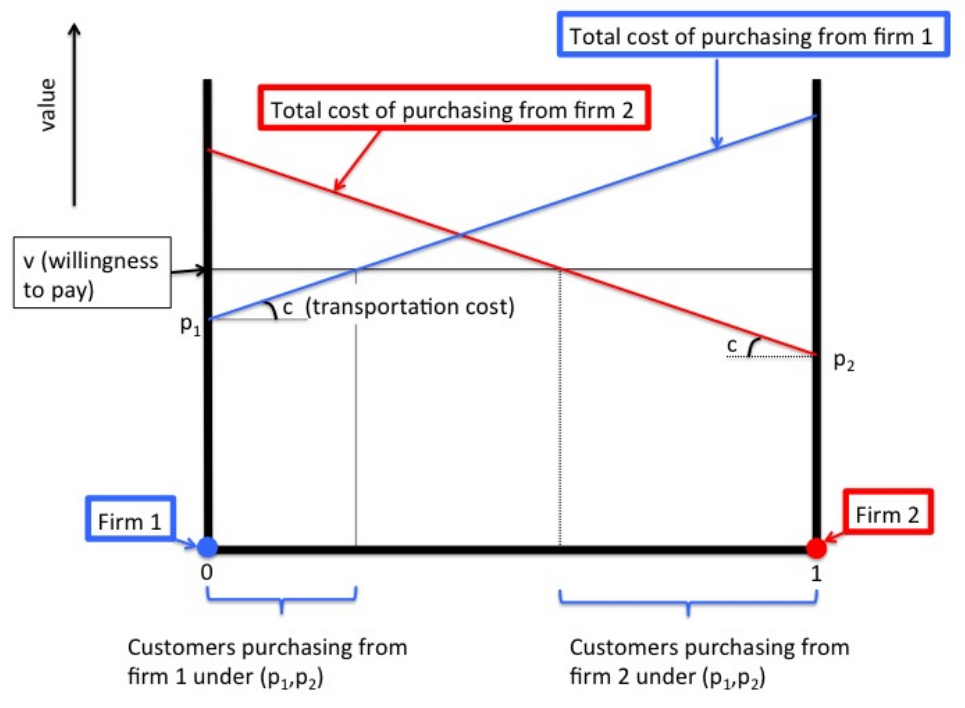

Figure 2: The component game for the Bertrand competition.

model with such a feature is the Hotelling's location model with price-setting firms. ${ }^{6}$ It is illustrated in Figure 2.

Figure 3 summarizes the main results. The figure shows the expected profits associated with the optimal trigger strategy equilibrium, joint profit maximization (full collusion), and the one-shot Nash equilibrium, all as a function of the level of product differentiation. In the model, consumers' transportation cost (c) relative to their value of the goods $(v)$ measures the degree of product differentiation. Note that, when $c / v=2 / 3=0.67$, each firm becomes a local monopolist and the Nash equilibrium coincides with the fully collusive outcome. Table 1 shows that the sustainable level of collusion varies from zero (when there is no differentiation) to higher levels as the differentiation increases.

\section{Model}

There is a unit mass of buyers uniformly distributed over $[0,1]$. Two firms $i=1,2$ are located at 0 and 1 , respectively. A buyer at location $s \in[0,1]$ receives payoff $v-c\left|s-s^{\prime}\right|-p$ if she buys from a firm at $s^{\prime}$ with price $p$. If the buyer does not buy, her payoff is 0 . When $c$ is high enough (i.e., $c>\frac{2}{3} v$ ), each firm becomes a

\footnotetext{
${ }^{6}$ An alternative would be to assume that firm $i$ 's demand is determined by $Q_{i}=a-b p_{i}+c p_{-i}$ under prices $\left(p_{i}, p_{-i}\right)$ for some constants $a, b, c>0$, but this specification does not nest the case of no differentiation as a special case.
} 


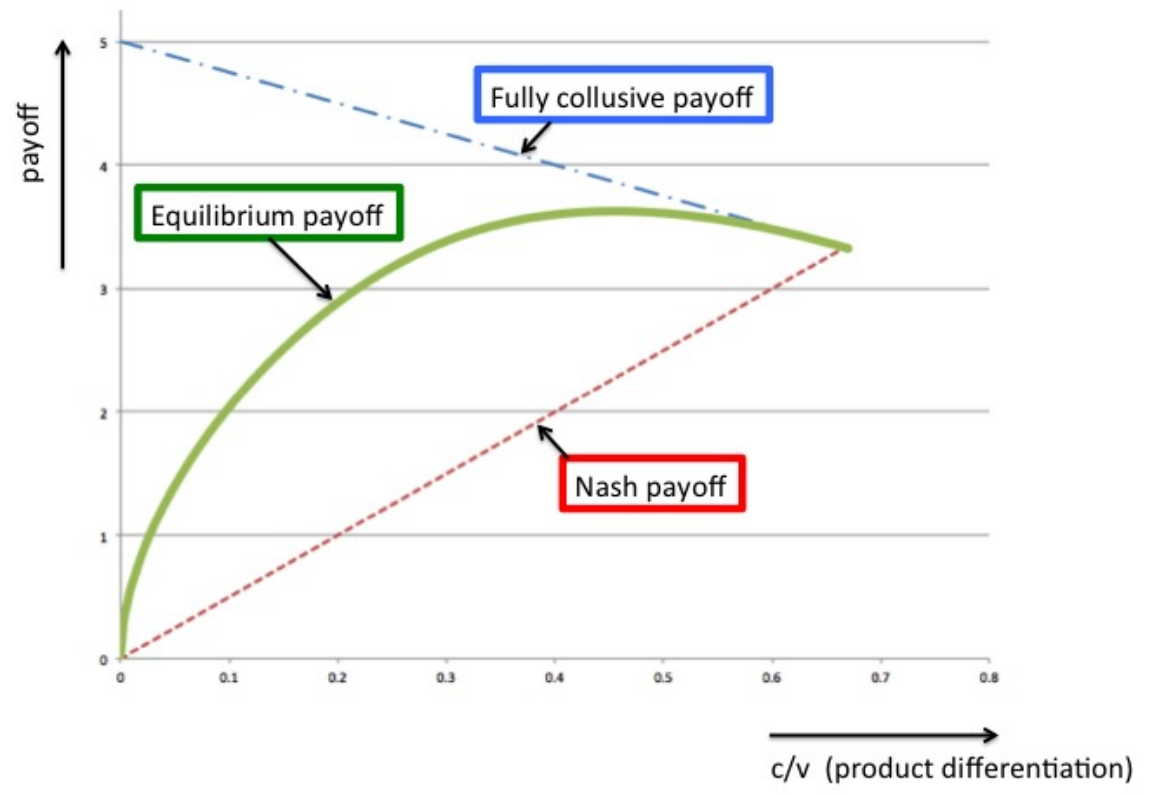

Figure 3: The expected payoffs under the optimal trigger strategy equilibrium plan for the Bertrand competition game: $v=10$.

local monopolist and the joint profit maximization is achieved by the one-shot Nash equilibrium. Hence, we focus on the non-trivial case $c \in\left[0, \frac{2}{3} v\right)$.

Each buyer can purchase one unit at most, and decides her purchase behavior to maximize the payoff. ${ }^{7}$ Each firm's marginal cost is normalized at 0 . The payoff function for firm $i$ is therefore given by:

$$
\pi_{i}\left(p_{i}, p_{-i}\right)=p_{i} \times\left(\text { market share } \operatorname{under}\left(p_{i}, p_{-i}\right)\right)
$$

\section{Differential equations}

It is straightforward to show that $p^{N}=c$ and $\pi^{N}=\frac{c}{2}$ hold in the Nash equilibrium, the fully collusive price is $p^{*}=v-\frac{c}{2}$, and the symmetric payoff function is $\pi(p)=\frac{p}{2} .{ }^{8}$ The differential equation depends on the gain from deviation $d(p)$, and it takes on two

\footnotetext{
${ }^{7}$ If a buyer is indifferent between purchasing and not, she makes a purchase. If all buyers are indifferent between the two firms, then the firms equally split the market share. If a buyer is indifferent between purchasing from two firms, she mixes between them with equal probability.

${ }^{8}$ We provide a detailed explanation for these values in the Appendix.
} 


\begin{tabular}{|c|c|c|c|c|c|c|}
\hline Degree of product differentiation $\left(h=\frac{c}{v}\right)$ & 0 & .1 & .2 & .3 & .5 & $.6 \dot{6}$ \\
\hline $\begin{array}{c}\text { Expected payoff } \\
\text { Fully collusive payoff }\end{array}$ & 0 & .429 & .641 & .797 & .963 & 1 \\
\hline$\frac{\text { Expected payoff - Nash payoff }}{\text { Fully collusive payoff - Nash payoff }}$ & 0 & .362 & .539 & .686 & .889 & 1 \\
\hline
\end{tabular}

Table 1: Degrees of product differentiation and cooperation (the right-bottom entry (i.e., 1 ) is the limit value as $h \uparrow \frac{2}{3}$ ) when the horizon is long enough.

functional forms for the following reasons. First, if $c$ is high relative to the rival's price $p$, the static best response is to steal only a part of the buyers from the rival firm. Second, if $c$ is relatively low, the static best response is to steal all the customers. Those two cases correspond to two functional forms of $d(p)$. For a high $c$, only the first case arises, while for a low $c$ both cases can arise.

1. High product differentiation: $c \in\left(\frac{2}{7} v, \frac{2}{3} v\right)$.

In this case, partial stealing of the customers from the rival is the myopic best reply and $d(p)=\frac{(p-c)^{2}}{8 c}$ holds for all $p \in\left[p^{N}, p^{*}\right]=\left[c, v-\frac{c}{2}\right]$. A1-A6 are satisfied for this range of $p$ as well, so Theorem 1 implies that the optimal trigger strategy equilibrium exists and its plan is a solution to the following differential equation:

$$
\frac{d p}{d t}=f(p)=\lambda \frac{p+3 c}{2}
$$

Since $\left|\frac{1}{f(p)}\right|<\infty$ holds for all $p \in\left(p^{N}, p^{*}\right]$ and $\lim _{p \downarrow c}\left|\frac{1}{f(p)}\right|<\infty$, Lemma 1 implies that the optimal trigger strategy equilibrium is nontrivial.

2. Low product differentiation: $c \in\left(0, \frac{2}{7} v\right]$.

In this case, the myopic best reply is full stealing of customers if the rival's price is above $\hat{p}:=3 c$, and a partial stealing is optimal otherwise. Hence, the functional form of the gain from deviation changes at $\hat{p}$;

$$
d(p)= \begin{cases}\frac{(p-c)^{2}}{8 c} & \text { if } \quad p \leq \hat{p} \\ \frac{p}{2}-c . & \text { if } \hat{p} \leq p\end{cases}
$$

Assumptions A1-A6 are satisfied for $p \in\left[p^{N}, p^{*}\right]=\left[c, v-\frac{c}{2}\right]{ }^{9}$ so Theorem 1

\footnotetext{
${ }^{9}$ Since $\lim _{p} \nearrow \hat{p} \frac{d(p)-d(\hat{p})}{p-\hat{p}}=\frac{1}{2}=\lim _{p} \backslash \hat{p} \frac{d(p)-d(\hat{p})}{p-\hat{p}}, d$ is differentiable at $p=\hat{p}$. Hence, A5 is satisfied. Note that $\pi_{i}$ is not differentiable at $\hat{p}-c$; however, A5 only requires the differentiability of $d$, so we can still apply our theorem.
} 
implies that the optimal trigger strategy equilibrium exists and its plan is a solution to the following differential equation:

$$
\frac{d p}{d t}=f(p)=\left\{\begin{array}{ll}
\lambda \frac{p+3 c}{2} & \text { if } p \leq \hat{p} \\
\lambda(2 p-3 c) . & \text { if } \hat{p} \leq p
\end{array} .10\right.
$$

Since $\frac{1}{f(p)}<\infty$ holds for all $p \in\left(p^{N}, p^{*}\right]$ and $\lim _{p \downarrow c}\left|\frac{1}{f(p)}\right|<\infty$, Lemma 1 implies that the optimal trigger strategy equilibrium is nontrivial.

\section{No product differentiation: $c=0$.}

In this case, an infinitesimal price-cut can steal the entire unit mass of buyers. Thus, the supremum payoff from deviating from the price profile $(p, p)$ is $p \times 1=$ $p$. This implies that $d(p)=p-\frac{p}{2}=\frac{p}{2} \cdot{ }^{11}$ A1-A2 and A4-A6 are satisfied for $p \in\left[p^{N}, p^{*}\right]$ (see footnote 11 regarding A3). Comparing the gain from deviation with the size of punishment, we have:

$$
\liminf _{p \downarrow p^{N}} \frac{d(p)}{\pi(p)-\pi^{N}}=\liminf _{p \downarrow p^{N}} \frac{\frac{p}{2}}{\frac{p}{2}-0}=1>0 .
$$

Hence, Part 3 of Theorem 1 implies that no cooperation is sustained by the trigger strategy when there is no product differentiation.

\section{Summary and comparative statics}

Overall, we obtain a conclusion that a nontrivial collusive plan exists if and only if there is a product differentiation. The intuition is as follows. If there is no product differentiation, each firm can steal the entire profit of the rival firm by an infinitesimal

\footnotetext{
${ }^{10}$ From this differential equation, we can compute the optimal plan. Specifically, we first use the differential equation $\frac{d p}{d t}=\lambda \frac{p+3 c}{2}$ for the region $\left[p^{N}, \hat{p}\right)$ with the initial condition at the deadline given by the time-price pair $(0, c)\left(c\right.$ is the Nash price $\left.p^{N}\right)$. Then, we consider the differential equation $\frac{d p}{d t}=\lambda(2 p-3 c)$ for the region $\left[\hat{p}, p^{*}\right]$ with the initial condition given by the time-price pair $(t(\hat{p}), \hat{p})$, where we define $t(\hat{p}):=\lim _{p} \nearrow_{\hat{p}} t(p)$ with $t(p)$ being the time at which the solution to the first differential equation is at price $p$. Note that, since the Finite Time Condition holds with $d(p)=\frac{(p-c)^{2}}{8 c}, t(\hat{p})$ is finite.

${ }^{11}$ Technically speaking, $d$ is not well-defined in the formula in A3 because there is no best response. In this paragraph, we use a modified definition of $d$ in which we replace "max" in the definition with "sup." For a similar reason, $\pi_{1}\left(a_{1}, a_{2}\right)$ is not continuous, which is again a violation of A3. However, the proof of Theorem 1 only uses the fact that $\pi(x)$ is continuous and $d(x)=\sup _{a_{1}} \pi_{1}\left(a_{1}, a\right)-\pi_{1}(a, a)$ exists and is continuous. These conditions are satisfied in the case of $c=0$ here, so the results in Theorem 1 still go through.
} 
price-cut, whenever the current price is strictly higher than the marginal cost (which is 0 in this example). This is because all buyers switch to the deviating firm. Hence, if the current price $p$ is not equal to the Nash price $p^{N}=c=0$, the gain from deviation $\left(d(p)=\frac{p}{2}\right)$ is of the same order in magnitude as the gain from cooperation $\left(\pi(p)-\pi^{N}=\frac{p}{2}\right)$, however close $p$ is to 0 . This makes cooperation impossible. If there is a product differentiation, however, only a small fraction of buyers switch to the deviating firm as a result of a marginal price cut. As a result, the gain from deviation $\left(d(p)=\frac{(p-c)^{2}}{8 c}\right)$ near the Nash price $\left(p^{N}=c\right)$ is of an order of magnitude smaller than the gain from cooperation $\left(\pi(p)-\pi^{N}=\frac{p-c}{2}\right)$ and this makes cooperation possible.

The differential equations in cases 1 and 2 above have closed-form solutions, and a formal description of the optimal trigger strategy equilibrium plan is described in the following:

Proposition 3. In the price competition revision game, the optimal trigger strategy equilibrium plan, $\bar{p}(t)$, is characterized as follows:

1. If $c \in\left(\frac{2}{7} v, \frac{2}{3} v\right)$,

$$
\bar{p}(t)=\left\{\begin{array}{ll}
c\left(4 e^{\lambda \frac{t}{2}}-3\right) & \text { if } t<t\left(p^{*}\right) \\
p^{*}=v-\frac{c}{2} & \text { if } t\left(p^{*}\right) \leq t
\end{array},\right.
$$

where $t\left(p^{*}\right)=\frac{2}{\lambda} \ln \left(\frac{v}{4 c}+\frac{5}{8}\right)$ is the time to achieve fully collusive price $p^{*}$.

2. If $c \in\left(0, \frac{2}{7} v\right]$,

$$
\bar{p}(t)= \begin{cases}c\left(4 e^{\lambda \frac{t}{2}}-3\right) & \text { if } t<\widehat{t} \\ c\left(\frac{8}{27} e^{2 \lambda t}+\frac{3}{2}\right) & \text { if } \widehat{t} \leq t<t\left(p^{*}\right) \\ p^{*}=v-\frac{c}{2} & \text { if } t\left(p^{*}\right) \leq t\end{cases}
$$

where $\widehat{t}=\frac{2}{\lambda} \ln \left(\frac{3}{2}\right)$ and $t\left(p^{*}\right)=\frac{3}{2 \lambda} \ln \left(\frac{3}{2}\right)+\frac{1}{2 \lambda} \ln \left(\frac{v}{c}-2\right)$.

3. If $c=0, \bar{p}(t)=0$ for all $t$.

The parameter $\widehat{t}$ in Case 2 is the time to achieve the critical price $\hat{p}$ in (4), where the functional form of the gain from deviation (and therefore that of the differential equation) changes. The optimal plans are depicted in Figure 4 for $v=10$ and $c=0,1,2,3$, and 5 . 


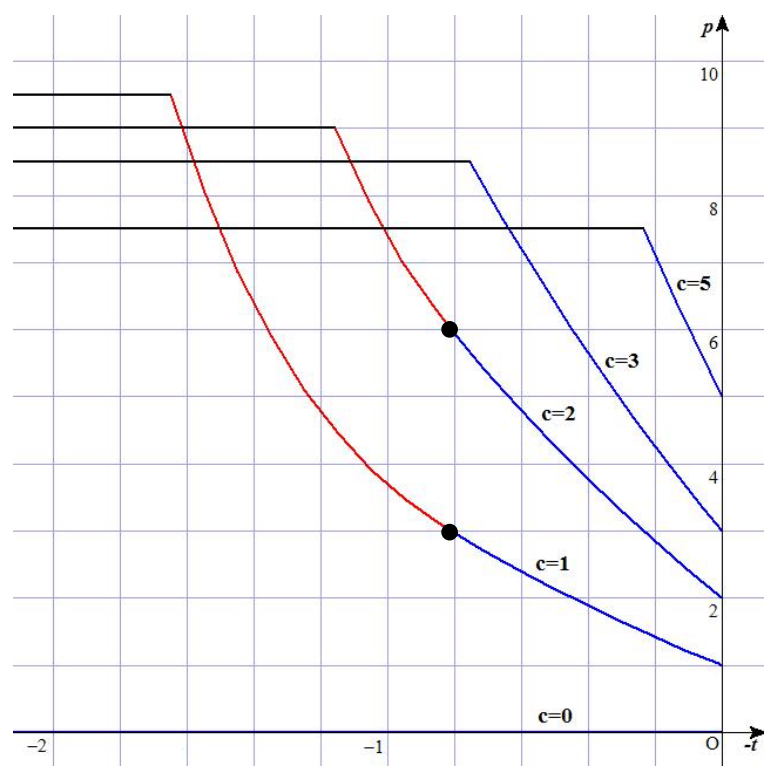

Figure 4: The optimal trigger strategy equilibrium plan $p(t)$ for the Bertrand competition game: $\lambda=1, v=10$. The black dots represent the time-price pairs $(\hat{t}, \hat{p})$ at which the two paths are pasted (cf. footnote 10).

As $c$ deceases, the Nash price $p^{N}$ decreases and the fully collusive price $p^{*}$ increases. The optimal plans are the curves connecting these two prices. Note that the optimal plan is the solution (the blue curve) to a single differential equation (3) when $c$ is high $(c=5,3)$. In contrast, when $c$ is low $(c=2,1)$, the optimal plan consists of the solutions to the two differential equations in (4) pasted together at the critical price level $\hat{p}:=3 c$ (the blue and red curves pasted at the black dots). The figure shows that, as the degree of product differentiation goes down to zero, the expected number of price revisions increases. This can be seen from the fact that the optimal price path departs from the fully collusive level farther away from the deadline when the degree of product differentiation is smaller (i.e., when $c$ is smaller). However, at the limit (i.e., when $c=0$ ), no price revision occurs even on the optimal trigger strategy equilibrium.

Let us calculate the expected payoff. So far, we have treated $c$ (transportation cost) as the degree of product differentiation. What really matters, however, is the magnitude of $c$ relative to $v$ (willingness to pay). Hence, we use $h:=\frac{c}{v}$ as the degree of product differentiation, and the expected payoff is characterized as follows. 
Corollary 2. In the price competition revision game, for any $h=c / v \in\left[0, \frac{2}{3}\right)$ and $T>t\left(p^{*}\right)$, the expected payoff under the optimal trigger strategy equilibrium is

$$
\left\{\begin{array}{ll}
v\left(\frac{5}{2} h-\frac{1}{4}\left(\frac{(8 h)^{2}}{2+5 h}\right)\right) & \text { if } h \in\left(\frac{2}{7}, \frac{2}{3}\right) \\
v\left(\frac{h}{2}+\frac{4 h}{9}\left(\left(\frac{3}{2 h}-3\right)^{\frac{1}{2}}\right)\right) & \text { if } h \in\left(0, \frac{2}{7}\right] \\
0 & \text { if } h=0
\end{array} .\right.
$$

The proof is given in Appendix B. Figure 3 at the beginning of this subsection shows the graph of the expected payoff and other benchmark payoffs. It shows that a significant degree of payoff improvement relative to the Nash equilibrium is achieved in the revision game.

The next corollary shows that the degree of collusion is increasing in the degree of product differentiation. Consider two measures of the degrees of collusion: First, $\bar{C}(h)$ is the expected payoff under the optimal trigger strategy equilibrium divided by the fully collusive payoff under $h$. Second, $\tilde{C}(h)$ is the expected payoff under the optimal trigger strategy equilibrium minus the Nash payoff, divided by the fully collusive payoff minus the Nash payoff under $h$. Note that $\tilde{C}(h)$ is a more conservative measure than $\bar{C}(h)$ because it measures the ratio of the payoff increment relative to the Nash payoff.

Corollary 3. The two measures of the degree of collusion are strictly increasing in the degree of product differentiation: $\bar{C}^{\prime}(h)>0$ and $\tilde{C}^{\prime}(h)>0$ if $T>t\left(p^{*}\right)$ under $h$.

Table 1 shows, for various degrees of product differentiation $h$, the values of $\bar{C}(h)$ and $\tilde{C}(h)$ when the horizon is long enough. ${ }^{12}$ As Corollary 3 predicts, those ratios are increasing in $h$. The table shows that the opportunities of revising prices can provide high levels of collusion, under reasonable degrees of product differentiation. For example, if $h=.5$, on average, a buyer's willingness to pay for the worse good is $71.4 \%$ of that of the preferred good. For such a degree of product differentiation, $96 \%$ of fully collusive payoffs can be achieved in the revision game. Even under the more conservative measure of cooperation, $89 \%$ of the increment in the expected profit relative to the Nash profit is achieved through the revision game.

\footnotetext{
${ }^{12}$ We can calculate these values since the ratios are the values derived in the corollary to $\pi^{*}=$ $\frac{1}{2}\left(v-\frac{c}{2}\right)$. We derive these ratios in Appendix B.
} 


\section{Appendix}

\section{A Detailed Analysis of the Bertrand Competition Component Game}

\section{Nash and fully collusive prices}

To understand the setting, first note that the symmetric Nash price is

$$
p^{N}=\left\{\begin{array}{ll}
c & \text { if } \quad c<\frac{2}{3} v \\
v-\frac{c}{2} & \text { if } \quad c \in\left[\frac{2}{3} v, v\right] . \\
\frac{v}{2} & \text { if } v<c
\end{array} .\right.
$$

There are three cases for the following reasons: First, when $c$ is very high $(v<c)$, the firms do not serve the buyers in $\left(\frac{v}{2 c}, 1-\frac{v}{2 c}\right)$, and each firm acts as if they were a monopoly. If $c$ is not too high $(c \leq v)$, a marginal price cut decreases the market share of the opponent, and the property of the Nash equilibrium depends on the relative sizes of $c$ and $v$.

To see this, it is easy to first consider the situation where $v$ is very high (specifically, the following discussion is for the case $c<\frac{2}{3} v$ ). In such a case, the Bertrand-type competition implies that the Nash price is low, while a Nash price is higher than the marginal cost (which is zero) because of product differentiation. The discrepancy between the marginal cost and the Nash price depends on the degree of product differentiation, and the Nash price is exactly $c$ in our linear-transportation-cost model. The reason that the Nash price is increasing in the transportation cost is that if the transportation cost is low, firms can decrease the price only a little bit to increase the market share significantly, so it is hard to sustain a high price. This feature of low transportation cost is going to be important in characterizing the optimal plan for the case with low transportation cost. We will be detailed on this in what follows.

When $v$ is small (specifically, the following discussion is for the case $c \in\left[\frac{2}{3} v, v\right]$ ), however, even if firms quote price $c$, not every customers make purchase. If this is the case, then each firm has an incentive to further cut their prices to induce all buyers to purchase. This is why the symmetric Nash price for low $c$ is exactly the value $v$ minus the transportation cost of the buyer at position $\frac{1}{2}$ (which is $c \times \frac{1}{2}$ ). ${ }^{13}$

\footnotetext{
${ }^{13}$ Note that there are multiple asymmetric equilibria in this case (i.e., when $c \in\left[\frac{2}{3} v, v\right]$ ) corre-
} 
Now, consider the fully collusive price:

$$
p^{*}=\left\{\begin{array}{ll}
v-\frac{c}{2} & \text { if } c \leq v \\
\frac{v}{2} & \text { if } v<c
\end{array} .\right.
$$

When $v<c$, the fully collusive price is $\frac{v}{2}$ for the same reason as for the Nash price. For the other case, it is optimal to divide the customer base in halves, inducing all customers to buy. That is, the fully collusive price is exactly at the price that makes the middle customer (at 0.5) indifferent between purchasing and not. Such a price increases as the transportation cost decreases, and this is why, for $c \leq v$, the fully collusive price increases as the product differentiation decreases.

In total, when $\frac{2}{3} v \leq c$, the unique Nash price profile coincides with the fully collusive profile, so there is no room for nontrivial cooperation. The intuition is that the cost of transportation is so high that a firm's marginal price cutting at the fully collusive price profile does not induce enough buyers to switch to the firm. For this reason, hereafter we assume that $c \in\left[0, \frac{2}{3} v\right)$. In this case, $\pi^{N}=\frac{c}{2}$ and $\pi(p)=\frac{p}{2}$.

\section{Gain from deviation}

The gain from deviation $d(p)$ depends on relative values of $c$ and $p$. If $c$ is high relative to $p$, then the static best response is not to steal all the buyers, but to tradeoff the decrease of the price and the increase of the customer base. This should be clear when the price is very close to the Nash price, which is when the best response is to set a price not too far from the Nash price, and cutting the price to steal all the customers is obviously suboptimal. On the other hand, if $c$ is relatively low, then an excessive price cut is not necessary to steal all the customer base, so the static best response is to drop the price to the one that is just enough to serve all the customers.

Since the range over which $p$ moves is dictated by the size of $v$, whether stealing all the customers can be a static best response on the equilibrium plan depends on the relative values of $c$ and $v$. Specifically, if $c \in\left(\frac{2}{7} v, \frac{2}{3} v\right)$, then there is no such possibility, and the static best response always solves the first order condition, and a calculation shows that $B R(p)=\frac{p+c}{2}$ and $d(p)=\frac{(p-c)^{2}}{8 c}$. If, however, $c \in\left(0, \frac{2}{7} v\right]$, then there is a possibility for the static best response to be stealing all customers when $p$ is high. In other words, the degree of differentiation is so small that when the opponent sponding to various possible splits of the market share. 


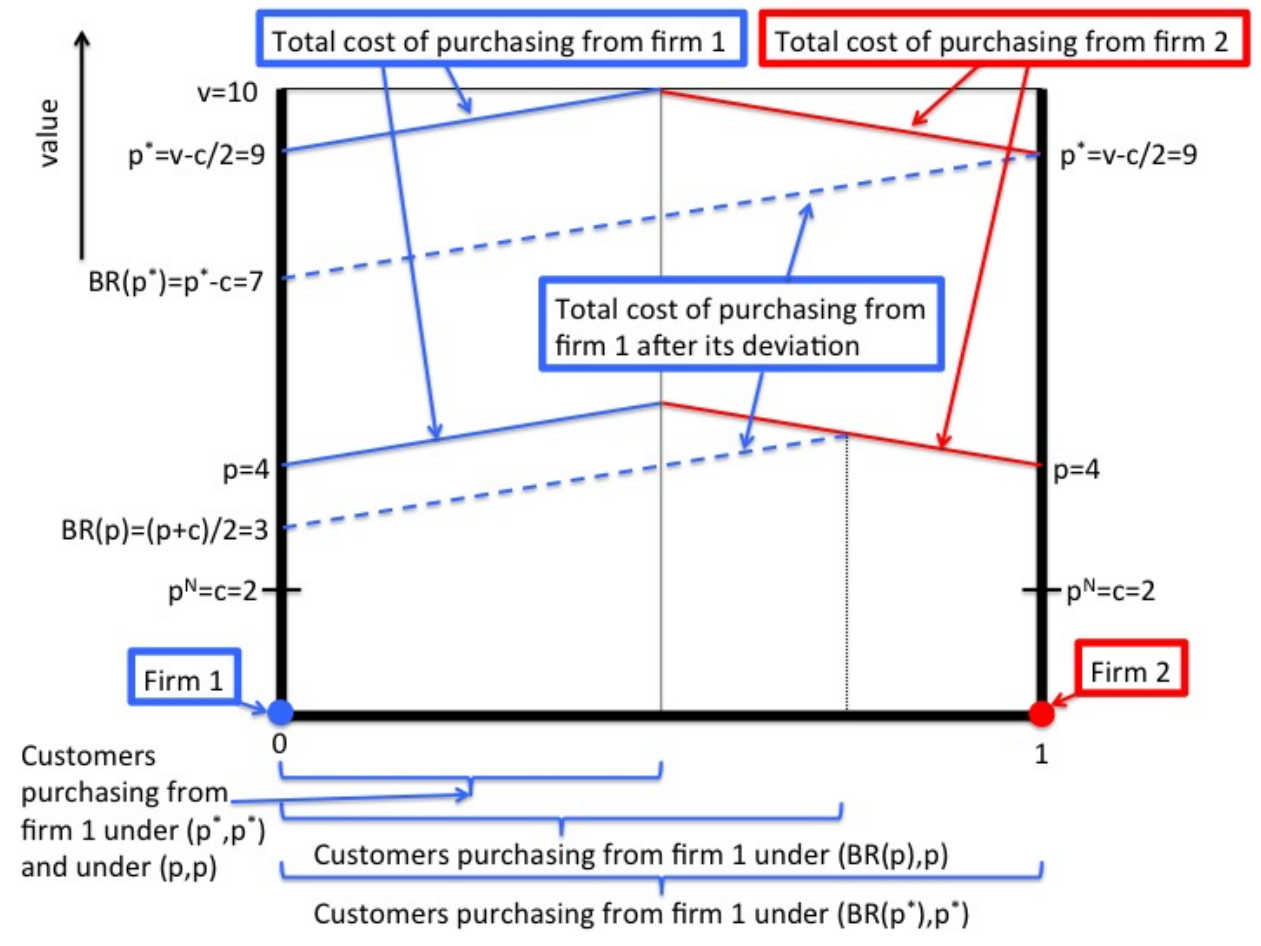

Figure 5: Low product differentiation: $v=10$ and $c=2$ (hence $\left.c \in\left(0, \frac{2}{7} v\right]\right)$ ).

sets a price close to the fully collusive price, the static best reply is to set a price just enough to attract all the buyers, that is, $B R(p)=p-c$ and hence $d(p)=\frac{p}{2}-c$. Figures 5 and 6 illustrate this difference by considering the cases with $c \in\left(0, \frac{2}{7} v\right]$ and $c \in\left(\frac{2}{7} v, \frac{2}{3} v\right)$, respectively. In the former case depicted in Figure 5, when the price is as high as the optimal price $p^{*}$, the static best response $B R\left(p^{*}\right)$ is such that the resulting price profile $\left(B R\left(p^{*}\right), p^{*}\right)$ gives the deviating firm (firm 1 in the figure) the market share of 1 . However, if the price is low (the case depicted as $p=4$ ), then the best response has to balance the increase in the market share and the decrease of the price. In the latter case depicted in Figure 6, even at the optimal price $p^{*}$, the best response $B R\left(p^{*}\right)$ does not give the deviating firm the market share of 1 . If the firm were to set a price such that it steals the whole market (to set the price $p=2.5$ in the example), then the firm's profit would be only as high as the Nash profit (i.e., both firms pricing at $p=p^{N}$ ). 


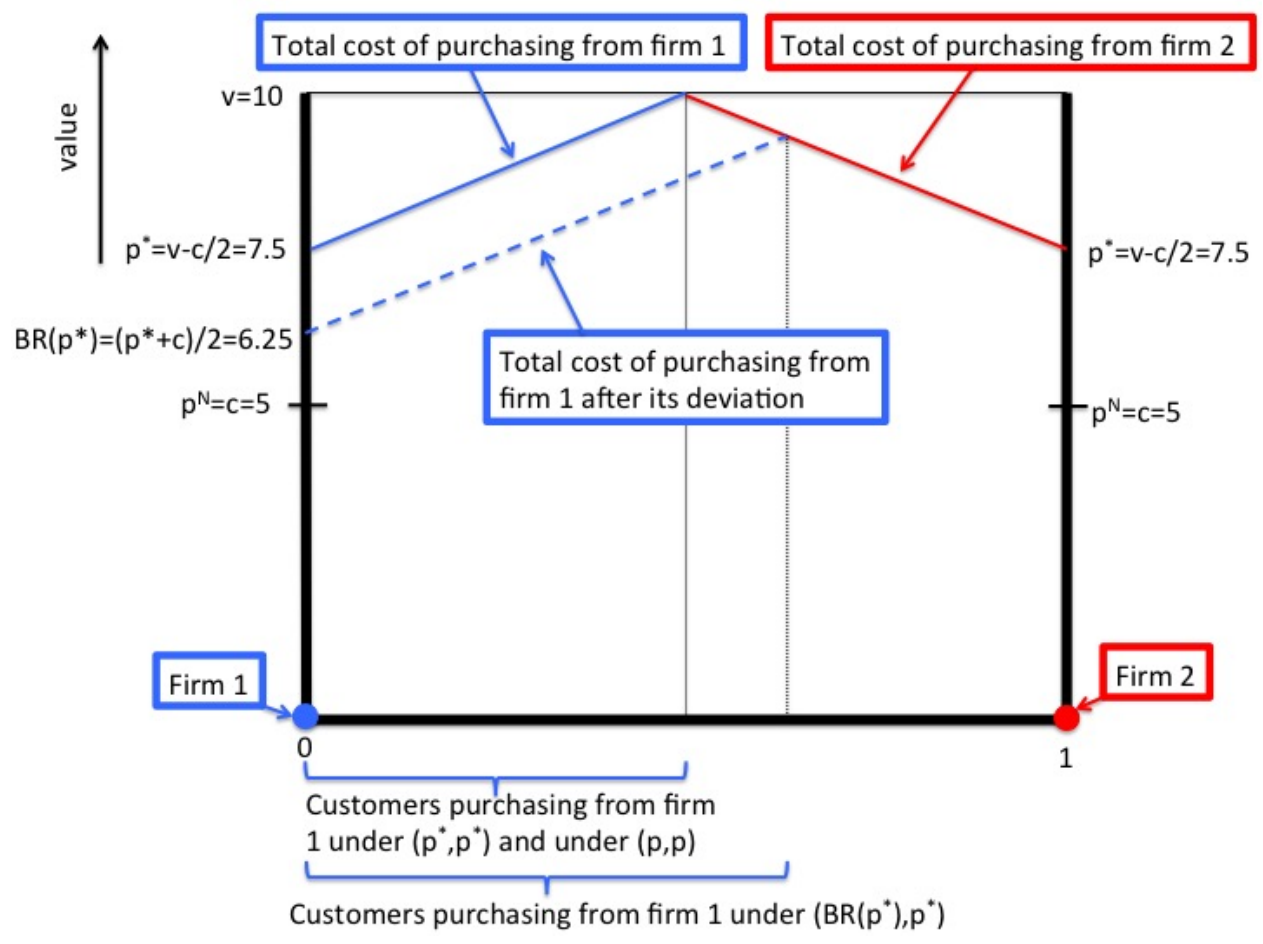

Figure 6: High product differentiation: $v=10$ and $c=5$ (hence $c \in\left(\frac{2}{7}, \frac{2}{3} v\right)$ ).

\section{B Expected Payoffs of the Bertrand Competition}

\section{The case of high product differentiation:}

Suppose $c \in\left(\frac{2}{7} v, \frac{2}{3} v\right)$. On the one hand, the expected payoff under the optimal trigger strategy equilibrium can be calculated as follows:

$$
\begin{aligned}
\int_{0}^{t\left(p^{*}\right)} \lambda e^{-\lambda t} \frac{\bar{p}(t)}{2} d t+e^{-\lambda t\left(p^{*}\right) \frac{p^{*}}{2}} & =\int_{0}^{\frac{2}{\lambda} \ln \left(\frac{v}{4 c}+\frac{5}{8}\right)} \lambda e^{-\lambda t} \frac{c\left(4 e^{\lambda \frac{t}{2}}-3\right)}{2} d t+e^{-\lambda \frac{2}{\lambda} \ln \left(\frac{v}{4 c}+\frac{5}{8}\right)} \frac{\left(v-\frac{c}{2}\right)}{2} \\
& =v\left(\frac{5}{2} h-\frac{1}{4}\left(\frac{(8 h)^{2}}{2+5 h}\right)\right) .
\end{aligned}
$$

On the other hand, the fully collusive payoff is $\frac{v-\frac{c}{2}}{2}=v \frac{1-\frac{h}{2}}{2}$. The ratio between these two values is:

$$
\bar{C}(h)=\frac{v\left(\frac{5}{2} h-\frac{1}{4}\left(\frac{(8 h)^{2}}{2+5 h}\right)\right)}{v \frac{1-\frac{h}{2}}{2}}=\frac{10 h(2+5 h)-64 h^{2}}{(2+5 h)(2-h)}=\frac{2 h(10-7 h)}{(2+5 h)(2-h)} .
$$


Differentiating this with respect to $h$, we obtain:

$$
\bar{C}^{\prime}(h)=\frac{(h+10)(2-3 h)}{(2+5 h)^{2}(2-h)^{2}} .
$$

Note that this is strictly positive whenever $h<\frac{2}{3}$. Thus, $\bar{C}(h)$ is strictly increasing in $h$.

Next, the ratio of the payoff increments is:

$$
\tilde{C}(h)=\frac{v\left(\frac{5}{2} h-\frac{1}{4}\left(\frac{(8 h)^{2}}{2+5 h}\right)\right)-\frac{c}{2}}{v \frac{1-\frac{h}{2}}{2}-\frac{c}{2}}=\frac{8 h}{5 h+2} .
$$

This is strictly increasing in $h$.

\section{The case of low product differentiation:}

Suppose $c \in\left(0, \frac{2}{7} v\right]$. The expected payoff under the optimal trigger strategy equilibrium can be calculated as follows:

$$
\begin{aligned}
& \int_{0}^{t^{1}} \lambda e^{-\lambda t} \frac{\bar{p}(t)}{2} d t+\int_{t^{1}}^{t^{2}} \lambda e^{-\lambda t} \frac{\bar{p}(t)}{2} d t+e^{-\lambda t^{2}} \frac{p^{*}}{2} \\
& =\int_{0}^{\frac{2}{\lambda} \ln \left(\frac{3}{2}\right)} \lambda e^{-\lambda t} \frac{c\left(4 e^{\lambda \frac{t}{2}}-3\right)}{2} d t+\int_{\frac{2}{\lambda} \ln \left(\frac{3}{2}\right)}^{\frac{3}{2 \lambda} \ln \left(\frac{3}{2}\right)+\frac{1}{2 \lambda} \ln \left(\frac{v}{c}-2\right)} \lambda e^{-\lambda t} \frac{c\left(\frac{8}{27} e^{2 \lambda t}+\frac{3}{2}\right)}{2} d t \\
& \quad+e^{-\lambda\left(\frac{3}{2 \lambda} \ln \left(\frac{3}{2}\right)+\frac{1}{2 \lambda} \ln \left(\frac{v}{c}-2\right)\right)} \frac{v-\frac{c}{2}}{2}=v\left(\frac{h}{2}+\frac{4 h}{9}\left(\frac{3}{2 h}-3\right)^{\frac{1}{2}}\right) .
\end{aligned}
$$

The fully collusive payoff can be calculated as before, and thus the ratio of the expected payoffs is:

$$
\bar{C}(h)=\frac{v\left(\frac{h}{2}+\frac{4 h}{9}\left(\left(\frac{3}{2 h}-3\right)^{\frac{1}{2}}\right)\right)}{\frac{v\left(1-\frac{h}{2}\right)}{2}}=\frac{2 h\left(1+\frac{8 h}{9} \sqrt{\frac{3}{2 h}(1-2 h)}\right)}{2-h} .
$$

Differentiating, we get

$$
\bar{C}^{\prime}(h)=\frac{4 \sqrt{3}\left(9 \sqrt{\frac{2(1-2 h)}{3 h}}+8 h^{2}-34 h+12\right)}{9(2-h)^{2} \sqrt{\frac{2(1-2 h)}{h}}} .
$$


Since $-34 h+12>0$ whenever $h \in\left(0, \frac{2}{7}\right)$, this is strictly positive for all $h \in\left(0, \frac{2}{7}\right)$. Hence, $\bar{C}(h)$ is strictly increasing in $h$.

Next, the ratio of the payoff increments is:

$$
\tilde{C}(h)=\frac{v\left(\frac{h}{2}+\frac{4 h}{9}\left(\left(\frac{3}{2 h}-3\right)^{\frac{1}{2}}\right)\right)-\frac{c}{2}}{\frac{v\left(1-\frac{h}{2}\right)}{2}-\frac{c}{2}}=\frac{16 \sqrt{3 h(1-2 h)}}{9 \sqrt{2}(2-3 h)} .
$$

Differentiating, we get:

$$
\tilde{C}^{\prime}(h)=\frac{8 \sqrt{3}(2-5 h)}{9(2-3 h)^{2} \sqrt{2 h(1-2 h)}} .
$$

This is strictly positive whenever $h \in\left(0, \frac{2}{7}\right)$. Hence, $\tilde{C}(h)$ is strictly increasing in $h$.

\section{References}

[1] Kamada Y. and M. Kandori (2017a): "Revision Games, Part I: Theory," mimeo.

[2] Kamada Y. and M. Kandori (2017b): "Revision Games, Part II: Applications and Robustness," mimeo.

[3] Kamada Y. and M. Kandori (2019): "Revision Games," mimeo.

[4] Sherstyuk, K., N. Tarui, and T. Saijo (2013): "Payment Schemes in InfiniteHorizon Experimental Games," Experimental Economics, 16(1): 125-153. 